\title{
L-NAME Reduces Infarct Volume in a Filament Model of Transient Middle Cerebral Artery Occlusion in the Rat Pup
}

\author{
STEPHEN ASHWAL, DANIEL J. COLE, SUZZANNE OSBORNE, TERRILL N. OSBORNE, AND \\ WILLIAM J. PEARCE \\ Departments of Pediatrics [S.A.], Anesthesiology [D.J.C., T.N.O., S.O.], and Physiology, Division of \\ Perinatal Biology [W.J.P.], Loma Linda University School of Medicine, Loma Linda, California 92354
}

\section{ABSTRACT}

The importance of nitric oxide (NO) during focal cerebral ischemia remains controversial as studies have suggested both a neurotoxic and neuroprotective role. In the $7 \mathrm{~d}$ old rat pup, $N^{\mathrm{G}}$-nitro-L-arginine, a nitric oxide synthase inhibitor, reduced infarct volume in a model of unilateral carotid ligation with 2.5 h exposure to $8 \% \mathrm{O}_{2}$. The current study examined whether $\mathrm{NO}$ is neurotoxic in a filament model of transient middle cerebral artery occlusion (MCAO) in the 14-18-d-old rat pup. We developed a reproducible filament model of transient MCAO in 1418-d-old spontaneously hypertensive rats $(35 \mathrm{~g})$ by passing a no. 6-0 $(0.07-\mathrm{mm})$ nylon filament via the carotid artery to occlude the middle cerebral artery for $4 \mathrm{~h}$ under normoxic conditions. After filament removal and reperfusion for $24 \mathrm{~h}$, we determined infarct volume using the mitochondrial stain 2,3,5-triphenyltetrazolium chloride. NO synthesis was inhibited using $N^{\mathrm{G}}$-nitro-Larginine methyl ester (L-NAME) at a dose of $3 \mathrm{mg} / \mathrm{kg}$, intraperitoneally, $1 \mathrm{~h}$ before MCAO. We measured infarct volume in control $(n=7)$ and L-NAME $(n=7)$ groups. L-NAME reduced infarct volume by $55 \%(p<0.01)$. In the control group, infarct volume $\left(180 \pm 29 \mathrm{~mm}^{3}\right)$ averaged $49 \pm 7 \%$ of the left hemisphere $\left(359 \pm 16 \mathrm{~mm}^{3}\right)$. In the L-NAME-treated group, infarct volume $\left(77 \pm 19 \mathrm{~mm}^{3}\right)$ was $22 \pm 5 \%$ of the left hemispheric volume $\left(344 \pm 2 \mathrm{~mm}^{3}\right)$. These findings support earlier studies that used models of neonatal hypoxic-ischemic brain injury and suggest a neurotoxic role of NO. They extend these observations by demonstrating a significant reduction in infarct volume in a stroke model in the immature rat pup. (Pediatr Res 38: 652-656, 1995)
CBF, cerebral blood flow
L-NAME, $N^{\mathrm{G}}$-nitro-L-arginine methyl ester
MCAO, middle cerebral artery occlusion
NO, nitric oxide
NOS, NO synthase
SHR, spontaneously hypertensive rat
TTC, 2,3,5-triphenyltetrazolium chloride
NMDA, $N$-methyl-D-aspartic acid

Abbreviations
Hypoxic-ischemic injury of the nervous system has been attributed, in part, to the neuronal release of excitatory amino acids. Recent studies suggest that this type of injury involves glutamate-induced neurotoxicity which might, to some degree, be mediated by NO (1-3). However, the role of NO remains controversial as there are now more than a dozen studies since 1991 which suggest either a neurotoxic or neuroprotective role of NO during ischemia (4-7). Endothelial NO release during ischemia might be beneficial by increasing CBF; alternatively, neuronal NO release during ischemia or reperfusion might be neurotoxic.

The role of hypoxic-ischemic excitotoxic neonatal cerebral injury has also received increasing attention. Demonstration of the role of glutamate release and NMDA receptor activation

Received November 3, 1994; accepted May 30, 1995.

Correspondence: Stephen Ashwal, M.D., Department of Pediatrics, West Hall, 11262 Campus Street, Loma Linda, CA 92354. during injury is now well established (8). Of interest is the observation that neonatal hypoxic-ischemic injury selectively spares NADPH-diaphorase-staining neurons that have been shown in adult models to synthesize NO $(2,9)$. In 7-d-old rat pups, inhibition of NOS with $N^{G}$-nitro-L-arginine $(50-100$ $\mathrm{mg} / \mathrm{kg}$ ) reduced hemispheric weight disparity in a model of left common carotid ligation with $8 \% \mathrm{O} 2$ and suggests that $\mathrm{NO}$ was neurotoxic (10). More recently, Hamada et al. (12) found a $77 \%$ reduction in cortical and an $88 \%$ reduction in striatal infarct volume using a similar model ( $N^{\mathrm{G}}$-nitro-L-arginine, 2 $\mathrm{mg} / \mathrm{kg}$ intraperitoneally) in 7-d-old rat pups with infarct volume determined by hemotoxylin and eosin staining.

We developed a reproducible transient MCAO stroke model in 14-18-d-old SHR pups and in the present study examined whether low dose NO inhibition with L-NAME $\left(\mathrm{N}^{\mathrm{G}}\right.$-nitro-Larginine methyl ester) affects cerebral infarct volume. We developed this model to reflect the physiologic events that occur during stroke due to cerebrovascular occlusion (11). 
With this filament model of MCAO there is minimal surgical trauma resulting in better preservation of cerebrovascular and endothelial function in contrast to doing a craniotomy that involves temporary surgical ligation of the middle cerebral artery, a procedure that is quite difficult. We used L-NAME, a nonspecific endothelial and neuronal NOS inhibitor administered intraperitoneally in low doses $(3.0 \mathrm{mg} / \mathrm{kg}) 1 \mathrm{~h}$ before the onset of 4-h MCAO and 24-h reperfusion because our previous studies demonstrated that this dose reduced infarct volume in the adult SHR by $55 \%$ (13). Although the 14-18-d-old rat pup model of transient MCAO may not be considered a true neonatal model, it is a model involving the immature brain that reflects changes occurring with development.

\section{METHODS}

Surgical preparation. We used 14-18-d-old SHR pups $(n=$ 14) whose weight averaged $35 \mathrm{~g}$. In our previous studies we used adult SHR (weight $=400 \mathrm{~g}$ ) because we have been able to create an extremely reproducible infarct using either a craniectomy ( $3 \mathrm{~h} \mathrm{MCAO}$ and $2 \mathrm{~h}$ reperfusion) or a filament model (2 h MCAO and $24 \mathrm{~h}$ reperfusion) $(13,14)$. In preliminary studies, we were able to produce a reliable infarct in these pups but animal size limitations precluded doing these studies in younger animals $(11,15)$.

SHR pups were anesthetized with $2 \%$ isoflurane (Forane) by mask and allowed to breathe spontaneously. End tidal isoflurane concentration (1.2\%) was determined with a PuritanBennett Datex Capnomac infrared anesthetic gas analyzer as previously reported $(16,17)$. Body temperature was maintained at $37^{\circ} \mathrm{C}$ throughout the experimental procedure, and the rat pups were maintained during the 24 -h period of reperfusion in a temperature-controlled environment at $95-100^{\circ} \mathrm{F}$. The temperatures were rechecked at $24 \mathrm{~h}$ and were similar in both control and experimental groups.

Establishment of reversible focal cerebral ischemia. SHR pups were placed in the supine position on the platform of a Zeiss stereoscopic microscope (model \#OMP1), and the left common carotid artery was exposed. The left external carotid artery was isolated and ligated. A tie was loosely placed around the internal carotid artery to control backflow, and a vascular clamp was placed on the common carotid artery $1 \mathrm{~cm}$ proximal to the bifurcation. A small incision was made in the external carotid artery close to the ligature and a no. $6-0(0.07 \mathrm{~mm})$ nylon filament passed proximally into the internal carotid artery to transiently occlude the middle cerebral artery. The filament was then sutured in place, the clamp on the common carotid artery removed, and the skin closed with 3-0 silk. The duration of this procedure was $20 \mathrm{~min}$. The pups were allowed to recover from anesthesia and were returned to their litters and maintained in a normoxic environment for $4 \mathrm{~h}$. After this period of normoxic MCAO, the pups were reanesthetized, the filament carefully removed, the external carotid artery ligated, and the wound closed. The pups were then returned to their litters for $24 \mathrm{~h}$ after which time they were killed so that infarct volumes could be determined.

Effect of $L$-NAME on cerebral infarct volume. We examined whether L-NAME ( $3 \mathrm{mg} / \mathrm{kg}$, intraperitoneally; $n=7$ ) given $60 \mathrm{~min}$ before 4-h MCAO and 24-h reperfusion reduced infarct volume compared with control animals $(n=7)$ given an equal volume of Ringer's lactate solution. The dose of LNAME and time period were chosen to ensure that the inhibitory effects of L-NAME on vascular and parenchymal NO synthesis were optimal during the period of occlusion and were based on previous pharmacokinetic studies that showed that the onset of the L-NAME effect begins within $30 \mathrm{~min}$ of infusion $(18,19)$.

Measurement of infarct volume. Neuropathologic evaluation of brain injury was accomplished using the histochemical stain TTC as previously reported by us and other investigators $(13,20-24)$. In normal brain, TTC is converted by mitochondrial oxidative enzymes to a red-formazan product, resulting in a deep red staining of brain parenchyma. Prolonged ischemia renders mitochondrial enzymes dysfunctional and causes failure of TTC conversion, resulting in a pale area in the brain. Several recent studies have shown good correlation in the measurement of infarct volume using TTC or conventional neuropathology at $24 \mathrm{~h}$ in the rat MCAO model $(22,23)$.

At the end of the 24-h reperfusion period, the pups were placed in an induction chamber, deeply anesthetized with 4.0-4.5\% Forane, after which a 24 gauge catheter (Insyte, Becton Dickinson) was inserted into the left ventricle, and a $2 \%$ TTC solution prewarmed to $37^{\circ} \mathrm{C}$ in isotonic saline was infused $(4 \mathrm{~mL} / \mathrm{min})$ for $8 \mathrm{~min}$ with effluent released through an incision in the right atrium. An additional $10 \mathrm{~min}$ for staining was allowed before fixation with a $10 \%$ buffered formalin/ $10 \%$ gluteraldehyde solution (1:1) using the same rate and volume of infusion. The brain was then removed, embedded in an egg albumin:gelatin medium, and mounted on a vibratome (Vibratome Series 1000; Technical Products International Inc., St. Louis, MO). Serial slices in $1.0-\mathrm{mm}$ increments spanning the area of middle cerebral artery distribution were cut, and seven standardized coronal brain sections were selected and photographed with color slide film (Ektachrome, Tungsten 160 ASA). The photographs were analyzed using a Drexel/ DUMAS Image Processing System and the area of deficient TTC staining determined manually by an observer blinded to treatment assignment. For each section, the volume of deficient staining and the volume of the entire hemisphere were determined, and a percent infarct volume was calculated. To estimate the percent infarct volume for the entire hemisphere, the infarct volumes and total hemisphere volumes were separately added, and the ratio was calculated. Because edema may alter the measurement of infarct volume, we corrected for this by using the previously published method of Swanson et al. (25).

Data analysis. The percent volume of infarction in control and L-NAME-treated animals was compared using a factorial one-way analysis of variance with two independent groups. Comparison of the seven sectional infarct volumes between control and L-NAME groups were analyzed with a repeated measures two-way analysis of variance. Throughout the text, results are given as the mean \pm the SEM, and $n$ refers to the number of animals. Unless otherwise specified, statistical significance was assumed at the $p<0.05$ level. 


\section{RESULTS}

L-NAME significantly reduced infarct volume by $55 \%(p<$ $0.01)$. In the control group $(n=7$; weight $=35.6 \pm 1.2 \mathrm{~g})$, infarct volume $\left(180 \pm 29 \mathrm{~mm}^{3}\right)$ averaged $49 \pm 7 \%$ of the left hemisphere $\left(359 \pm 16 \mathrm{~mm}^{3}\right)$. In the L-NAME-treated group $(n$ $=7$; weight $=35.5 \pm 1.4 \mathrm{~g})$, infarct volume $\left(77 \pm 19 \mathrm{~mm}^{3}\right)$ was $22 \pm 5 \%$ of the left hemispheric volume $\left(344 \pm 2 \mathrm{~mm}^{3}\right)$. In addition, the left hemisphere volume in the control group was $4.6 \%$ greater than that observed in the L-NAME group and provides additional supportive data that L-NAME reduced neurotoxicity. In Figure 1 a representative section of a control and L-NAME section are shown, demonstrating the volume of mitochondrial pallor that reflects focal ischemic injury. Figure 2 depicts the seven serial cross-sections from the SHR pup brain with the black shaded area indicating the mean volume of infarction for that section. Significant differences were observed between control and L-NAME groups for sections two to seven $(0.009<p>0.03 ; p$ value for section one $=0.12)$.
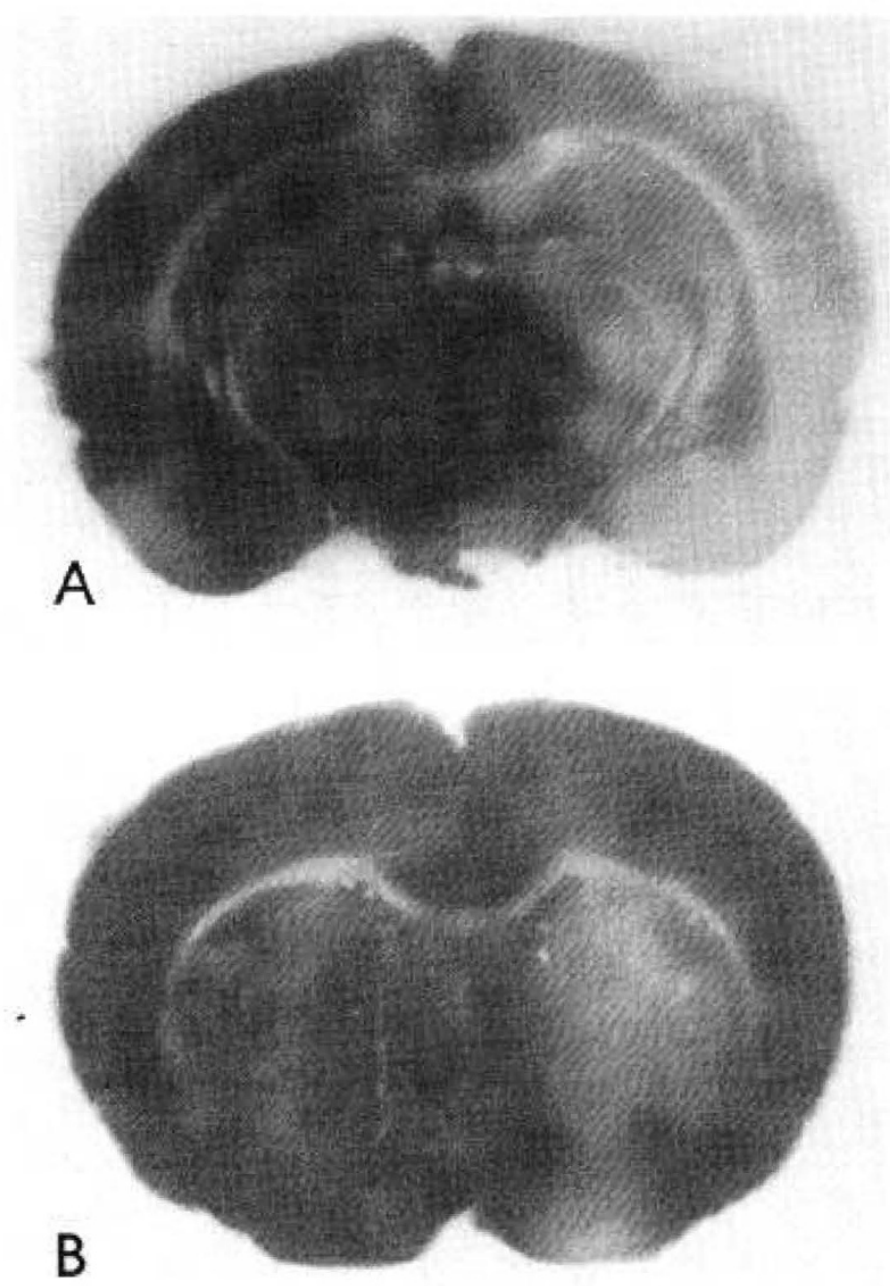

Figure 1. Coronal TTC-stained brain sections demonstrating an area of pallor indicating ischemic injury in the distribution of the middle cerebral artery from a control $(A)$ and a L-NAME-treated $(B)$ rat pup. The area of pallor includes cortical and caudoputamenal regions. The area of injury measured $68 \%$ of hemispheric area in the control section and $32 \%$ in the L-NAME section.

\section{DISCUSSION}

The present study demonstrates that inhibition of NOS using low dose L-NAME reduced infarct volume in a filament model of transient MCAO in the 14-18-d-old rat pup. These findings support previous studies that have suggested that NO may be neurotoxic in the developing nervous system $(10,12)$.

NO and models of focal cerebral ischemia. Studies examining the role of NO during focal cerebral ischemia have been difficult to interpret as the results have been conflicting (4-7). As yet, no unifying theory has been proposed that can reconcile these contradictory findings with the dual nature of NO as both a vasodilator and neurotoxin. Experimental variables such as the model used to create ischemia (permanent versus focal), the dose and specific NOS inhibitor administered, and the duration of MCAO and reperfusion have all affected the results and thus the interpretation of the role of NO. Studies by several investigators have suggested that NO release during ischemia might increase CBF $(4,5)$. Our recent studies (13) as well as those of Huang et al. (26) have provided convincing evidence that although NO release during focal ischemia is likely to increase $\mathrm{CBF}$ and potentially reduce infarct volume, the overall effect of NO is that of serious neurotoxicity.

Glutamate and NO neurotoxicity in the newborn. There is substantial evidence that glutamate toxicity may be important in the developing brain $(8,27,28)$. Marked elevation of extracellular glutamate and aspartate occur in the striatum and cortex during fetal lamb asphyxia and parallel the severity of asphyxia in a neonatal rat model of focal cerebral ischemia (29, 30). In addition, the neuropathologic progression after hypoxic-ischemic injury in the neonate is indistinguishable from that due to excitotoxic damage due to glutamate administration (31). It has also been shown that MK-801, a NMDA receptor antagonist, can block hypoxic-ischemic injury in the neonatal rat (28).

As glutamate and other excitatory neurotransmitters are suspected of playing a role in the neurotoxicity associated with hypoxic-ischemic brain injury, it has been suggested that NO might be one of the agents that mediates this response $(1,2)$. $\mathrm{NO}$ is formed in response to NMDA receptor activation by excitatory neurotransmitters such as glutamate (32). NOS inhibitors decrease brain NO production and also prevent NMDA neuronal toxicity in rat cortex, caudate-putamen, and hippocampal cell culture studies as well as in vitro in the hippocampal slice preparation and in vivo in the hippocampus (2, 33-35). Finally, NOS inhibition with L-NAME, has been shown to reduce infarct volume produced by NMDA receptor activation by $35 \%$ (36).

The relation between glutamate neurotoxicity and NO during development remains complex. Each of the glutamate receptor subtypes possess unique developmental neurotoxicity profiles (37). In the 7-d-old rat pup glutamate neurotoxicity is mediated primarily by NMDA and quisqualate/ $\alpha$-amino-3hydroxy-5-methyl-4-isoxazole propionic acid receptor activation in contrast to the adult rat where kainate is more neurotoxic. Moreover, glutamate susceptibility in different brain regions and neuronal populations changes significantly with development. In addition, both cytosolic and particulate forms 


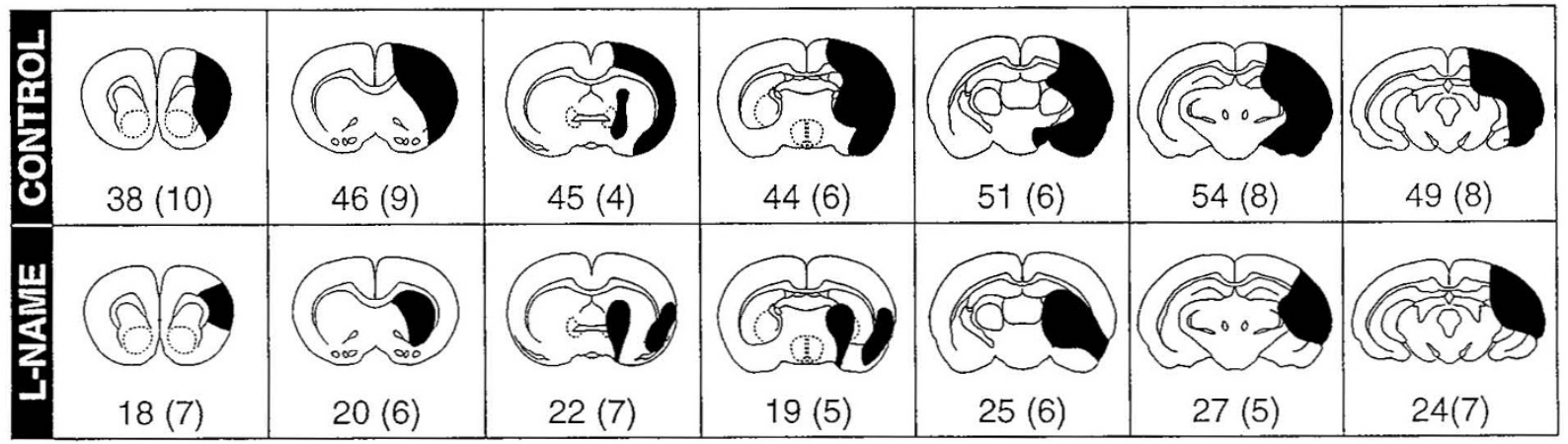

Figure 2. Seven representative cross-sections from the brain of control $(n=7)$ and L-NAME-treated $(n=7)$ groups are depicted from anterior (left; section one) to posterior (right; section seven). The darkened areas represent the mean percent volume of infarction for that group at that particular cross-sectional level. The distribution of infarct that is outlined was based on the results of our determination of the areas of infarction for each sectional level. The mean ( \pm SEM) percent volume of infarction are given under each section. L-NAME significantly reduced infarct volume in sections two to seven $(0.009<p>0.03 ; p$ value for section one $=0.12$ )

of NOS change with development $(38,39)$. In rat cerebrum, particulate NOS increases in the first postnatal week, then decreases and becomes almost undetectable in the adult. In contrast, cytosolic NOS increases somewhat in the rat cerebrum and about 8 -fold in the cerebellum over the same time frame. Irrespective of these complex developmental changes, the fact that NOS inhibition reduced infarct volumes in 7- (12) and 14-18-d-old rat pups (current study) suggests a relation between focal ischemic injury, NO synthesis and neurotoxicity.

NO neurotoxicity during focal cerebral ischemia in the developing brain. Several mechanisms have been suggested to explain NO-mediated neurotoxicity including free radical induced damage to proteins, DNA, and membrane lipids; direct inactivation of enzymes involved in mitochondrial respiration; and energy depletion subsequent to activation of poly-ADP ribose synthase after NO-dependent damage (7). The relative contributions of any of these mechanisms in either focal or global neonatal hypoxic-ischemic injury have yet to be investigated. Of interest is the observation that NOS expression (and presumably NO production) may play an important role in the regulation of axonal projections during development (40). This may be of particular importance during focal cerebral ischemia as recent studies have shown not only an increase in NO immediately after occlusion but a delayed more marked increase in NO production that peaks at $24 \mathrm{~h}$ and lasts for several days (41). Thus, NO neurotoxicity could persist long after the insult and might have a subtle but definite impact on neuronal and glial development.

A new model of stroke in the immature rat pup. The present study demonstrates the feasibility of adapting an adult model of reversible MCAO without craniectomy to the rat pup. The desire to develop a reliable rat pup model of reversible focal ischemia was based on the limitations of currently available models of focal cerebral ischemia in the newborn animal. The Rice-Vannucci model of unilateral carotid ligation with 2-3 h of exposure to $8 \% \mathrm{O}_{2}$ has been used in the rat to examine a variety of questions related to the pathogenesis of neonatal hypoxic-ischemic injury $(11,42)$. Although, the lesion created with this model is unilateral, it is primarily a model of a diffuse hypoxic-ischemic hemispheric insult rather than one due to focal occlusive cerebrovascular disease (e.g. neonatal stroke) as is the filament MCAO model.

Since 1986, several studies have been published describing filament models of reversible MCAO without craniectomy in the adult rat (43-46). Infarct volume (TTC) averaged $37.6 \%$ with permanent MCAO and $21.9 \%$ after $2 \mathrm{~h}$ and $25.7 \%$ after 4 $\mathrm{h}$ of reversible MCAO (45). A more recent study found that CBF was reduced to near constant values at 20,60 , and 120 min of occlusion and averaged $10 \%$ of controls in the area of irreversible infarction and $15-20 \%$ in the penumbral region (46). With 24-h permanent MCAO, infarct volume measured $70 \%$ in cortex and caudoputamen; with 1-h reversible MCAO infarct volume at $7 \mathrm{~d}$ measured $30-35 \%$.

The volume of infarct involving cortical as well as caudoputamenal regions that we observed in our 14-18-d-old rat pup model is similar to that noted in the above studies. Developing a newborn model of reversible MCAO without craniectomy was technically challenging. In contrast to adult rats that weighed $350-400 \mathrm{~g}, 14-18$-d-old rat pups average $35 \mathrm{~g}$. The filament diameter and length are substantially smaller than that used in adults. In these animals it is also difficult to cannulate the femoral artery for measurement of different physiologic variables or the femoral vein for drug infusions and intraperitoneal injections are usually necessary.

A limitation of this filament model is the age of the rat pups when a stroke can reliably be created. Unilateral carotid ligation with $8 \% \mathrm{O}_{2}$ can be accomplished in the rat pup after $7 \mathrm{~d}$ of age (42). In the filament model of MCAO without craniectomy, size limitations preclude performing this procedure before $14 \mathrm{~d}(35 \mathrm{~g})$. Thus, there are limitations in considering this a model of neonatal stroke as many biochemical, physiologic, and anatomical changes occur in the rat pup between $\mathrm{d} 7$ and d 14-18 $(38,39)$. These two models of cerebrovascular injury (i.e. unilateral carotid ligation with $8 \% \mathrm{O}_{2}$ versus normoxic filament MCAO), however, can be considered complementary as they examine two different types of cerebral insults (hypoxic-ischemic injury versus stroke) that occur in the neonatal period. The clinical relevance for developing a model of neonatal stroke has become apparent over the past decade as neuroimaging studies have convincingly demonstrated that 
such lesions are more common than previously recognized and account for serious neurologic morbidity. Although the incidence is uncertain, recent studies have suggested that neonatal stroke accounts for up to $20 \%$ of patients with neonatal seizures, and other investigators have found a $5 \%$ incidence of neonatal stroke at autopsy (47).

\section{CONCLUSIONS}

L-NAME reduced infarct volume by $55 \%$ in a filament model of transient focal cerebral ischemia in the SHR pup. These findings are similar in magnitude to that which we observed in the adult SHR using the same dose of L-NAME (13). They also support earlier studies that used models of neonatal hypoxic-ischemic brain injury and suggest a neurotoxic role for NO in the developing nervous system.

\section{REFERENCES}

1. Beckman J 1991 The double-edged role of nitric oxide in brain function and superoxide-mediated injury. J Dev Physiol 15:53-59

2. Dawson TM, VL Dawson, SH Snyder 1992 A novel neuronal messenger molecule in brain: the free radical, nitric oxide. Ann Neurol 32:197-311

3. Lipton SA, Rosenberg PA 1994 Excitatory amino acids as a final common pathway for neurologic disorders. N Engl J Med 33:613-622

4. Pelligrino DA 1993 Saying NO to cerebral infarction. J Neurosurg Anesthesiol 5:221-231

5. Iadecola C, Pelligrino, D, Moskowitz MA, Lassen NA 1994 Nitric oxide synthase inhibition and cerebrovascular regulation. J Cereb Blood Flow Metab 14:175-192

6. Dalkara T, Moskowitz MA 1994 The complex role of nitric oxide in the pathogenesis of focal cerebral ischemia. Brain Pathol 4:49-57

7. Dawson DA. 1994 Nitric oxide and focal cerebral ischemia: multiplicity of actions and diverse outcome. Cerebrovasc Brain Metab Rev 6:299-324

8. Rothman SM 1992 Excitotoxins: possible mechanism of action. Ann NY Acad Sci 648:132-139

9. Ferriero DH, Arcavi LJ, Sagar SM, McIntosh TK, Simon RP 1988 Selective sparing of NADPH-diaphorase neurons in neonatal hypoxia-ischemia. Ann Neurol 24:670676

10. Trifiletti R 1992 Neuroprotective effects of $N^{G}$-nitro-L-arginine in focal stroke in the 7-day-old rat. Eur J Pharmacol 218:197-198

11. Ashwal S, Cole DJ, Osborne TN, Osborne S, Pearce WJ 1995 A new model of neonatal stroke; reversible middle cerebral artery occlusion in the rat pup. Pediatr Neurol 12:191-196

12. Hamada Y, Hayakawa T, Hattori H, Mikawa H 1994 Inhibitors of nitric oxide synthesis reduces hypoxic-ischemic brain damage in the neonatal rat. Pediatr Res 35:10-14

13. Ashwal S, Cole DJ, Osborne TN, Pearce WJ 1994 Dual effects of L-NAME during transient focal cerebral ischemia in the spontaneously hypertensive rat. Am J Physiol 267:H276-H284

14. Cole DJ, Drummond JC, Patel PM, Katsuyama S 1994 Hemodilution during cerebral ischemia in rats: effects of a molecular hemoglobin solution on neurologic outcome and brain injury. Anesth Analg 51:S66

15. Ashwal S, Cole DJ, Osborne TN, Osborne S, Pearce WJ 1994 Nitric oxide inhibition reduces infarct volume during transient neonatal focal cerebral ischemia in the SHR. Ann Neurol 36:500.

16. Ashwal S, Cole DJ, Osborne TN, Pearce WJ 1993 Low dose L-NAME reduces infarct volume in the rat MCAO/REPERFUSION model. J Neurosurg Anesthesiol 5:241249

17. Cole DJ, Drummond JC, Ghazal EA, Shapiro HM 1990 A reversible component of cerebral injury as identified by the histochemical stain 2,3,5-triphenyltetrazolium chloride (TTC). Acta Neuropathol 80:152-155

18. Irikura K, Rosenblatt S, Kontos HA, Moskowitz MA 1993 The effect of nitric oxide synthase (NOS) inhibition on pial artery dilation produced by somatosensory stimulation and hypercapnea. J Cereb Blood Flow Metab 13(suppl):S132

19. Malinski T, Bailey F, Zhang ZG, Chopp M 1993 Nitric oxide measured by a porphyrinic microsensor in rat brain after transient middle cerebral artery occlusion. J Cereb Blood Flow Metab 13:355-358
20. Bederson J, Pitts L, Germano S, Nishimura M, Davis R, Bartkowski H 1986 Evaluation of 2,3,5-triphenyltetrazolium chloride as a stain for detection and quantification of experimental cerebral infarction in rats. Stroke 17:1304-1308

21. Park C, Mendelow A, Graham D, McCulloch J, Teasdale G 1988 Correlation of triphenyltetrazolium chloride perfusion staining with conventional neurohistology in the detection of early brain ischaemia. Neuropathol Appl Neurobiol 14:289-298

22. Hatfield RH, Mendelow AD, Perry RH, Alvarez LM, Modha P 1991 Triphenyltetrazolium chloride (TTC) as a marker for ischaemic changes in rat brain following permanent middle cerebral artery occlusion. Neuropathol Appl Neurobiol 17:61-67

23. Isayama K, Pitts LH, Nishimura MC 1991 Evaluation of 2:3,5-triphenyltetrazolium chloride staining to delineate rat bran infarcts. Stroke 22:1394-1398

24. Saeed D, Goetzman BW, Gospe SM Jr 1993 Brain injury and protective effects of hypothermia using triphenyltetrazolium chloride in neonatal rat. Pediatr Neurol 9:263-267

25. Swanson RA, Morton MT, Tsao-Wu G, Savalos RA, Davidson C, Sharp FR 1990 A semiautomated method for measuring brain infarct volume. J Cereb Blood Flow Metab 10:290-293

26. Huang Z, Huang PL, Panahian N, Dalkara T, Fishman MC, Moskowitz MA 1994 Effects of cerebral ischemia in mice deficient in neuronal nitric oxide synthase. Science 265:1883-1885

27. Hattori H, Wasterlain CG 1990 Excitatory amino acids in the developing brain ontogeny, plasticity, and excitotoxicty. Pediatr Neurol 6:219-228

28. McDonald JW, Silverstein F, Johnston MV 1989 Neuroprotective effects of MK-801, TCP, PCP and CPP against $N$-methyL-D-aspartate induced neurotoxicity in an in vivo perinatal rat model. Brain Res 490:33-40

29. Hagberg H, Andersson P, Kjellmer I, Thiringer K, Thordstein M 1987 Extracellular overflow of glutamate, aspartate, GABA, and taurine in the cortex and basal ganglia of fetal lambs during hypoxia-ischemia. Neurosci Lett 78:311-317

30. Silverstein FS, Naik B, Simpson J. 1991 Hypoxia-ischemia stimulates hippocampal glutamate efflux in perinatal rat brain: an in vivo microdialysis study. Pediatr Res 30:587-590

31. Ikonomidou C, Mosinger JL, Salles KS, Labruyere J, Olney JW 1989 Sensitivity of the developing rat brain to hypobaric/ischemic damage parallels sensitivity to $\mathrm{N}$ methyL-aspartate neurotoxicity. J Neurosci 9:2809-2818

32. Garthwaite J 1991 Glutamate, nitric oxide and celt-cell signaling in the nervous system. Trends Neurosci 14:60-67

33. East SJ, Garthwaite J 1990 Nanomolar $N^{G}$-nitroarginine NMDA-induced cyclic GMP formation in rat cerebellum. Eur J Pharmacol 184:311-313

34. Dawson VL, Dawson TM, London ED, Bredt DS, Snyder SH 1991 Nitric oxide mediates glutamate neurotoxicity in primary cortical cultures. Proc Natl Acad Sci USA 88:6368-6371

35. Moncada C, Lekieffre D, Arvin B, Meldrum B 1992 Effect of NO synthase inhibition on NMDA-and ischaemia-induced hippocampal lesions. Neuroreport 3:530-532

36. Buisson A, Margaill I, Callebert J, Plotkine M, Boulu RG 1993 Mechanisms involved in the neuroprotective activity of a nitric oxide synthase inhibitor during focal cerebral ischemia. J Neurochem 61:690-696

37. McDonald JW, Johnston MV 1990 Physiological and pathophysiological roles of excitatory amino acids during central nervous system development. Brain Res Rev $15: 41-70$

38. Matsumoto T, Pollock JS, Nakane M, Forstermann U 1993 Developmental changes of cytosolic and particulate nitric oxide synthase in rat brain. Brain Res Dev Brain Res 73:199-203

39. Bredt DS, Snyder SH 1994 Transient nitric oxide synthase neurons in embryonic cerebral cortical plate, sensory ganglia, and olfactory epithelium. Neuron 13:301-313

40. Williams CV, Nordquist D, McLoon SC 1994 Correlation of nitric oxide synthase expression with changing patterns of axonal projections in the developing nervous system. J Neurosci 14:1746-1755

41. Zhang ZG, Chopp M, Pollock JS, Förstermann U. 1993 Cerebral endothelial nitric oxide synthase expression after focal cerebral ischemia in rats. Stroke 24:2016-2022

42. Rice J, Vannucci R, Brierly J 1981 The influence of immaturity on hypoxia-ischemic brain damage in the rat. Ann Neurol 9:131-141

43. Koizumi J, Yoshida Y, Nakazawa T, Ooneda G 1986 Experimental studies of ischemic brain edema. 1. A new experimental model of cerebral embolism in rats in which recirculation can be introduced in the ischemic area. Jpn $\mathrm{J}$ Stroke 8:1-8

44. Nagasawa H, Kogure K 1989 Correlation between cerebral blood flow and histologic changes in a new rat model of middle cerebral artery occlusion. Stroke 20:1037-1043

45. Longa EZ, Weinstein PR, Carlson S, Cummins R 1989 Reversible middle cerebral artery occlusion without craniectomy in rats. Stroke 20:84-91

46. Memezawa H, Minamisawa H, Smith ML, Siesjo BK 1992 Ischemic penumbra in a model of reversible middle cerebral artery occlusion in the rat. Exp Brain Res 89:67-78

47. Perlman JM, Rollins NK, Evans D 1994 Neonatal stroke: clinical characteristics and cerebral blood flow velocity measurements. Pediatr Neurol 11:281-284 